\title{
An aggressive approach leads to improved survival in hepatocellular carcinoma patients with portal vein tumor thrombus
}

\author{
De-Xin Lin • Qi-Yu Zhang $\cdot$ Xuan Li $\cdot$ Qi-Wen Ye • \\ Fen Lin $\cdot$ Lin-Li Li
}

Received: 5 February 2010/ Accepted: 9 March 2010/Published online: 26 March 2010

(c) The Author(s) 2010. This article is published with open access at Springerlink.com

\begin{abstract}
Purpose Many physicians express a relatively nihilistic approach to the treatment of hepatocellular carcinoma (HCC) with portal vein tumor thrombus (PVTT). Consensus among surgeons regarding the indications for an aggressive approach has not been reached. Current study was aimed to determine whether an aggressive approach, with an extended resection with thrombectomy and adjuvant therapy, would lead to an improved survival for HCC patients with PVTT. Methods A retrospective review of 116 HCC patients with PVTT admitted from 1996 to 2006 was conducted. Patients were divided into 2 time-period (TP) cohorts, of them, 51 cases in the first 5 years (TP1) and 65 in the last 5 years (TP2).

Results Surgical operations were performed on 68 patients. Twenty-one surgical resections were performed in TP1 and forty-seven in TP2. The extent of liver resections, as well as the frequency of thrombectomy, was greater in TP2 $(P=0.039)$. During both time-periods, an aggressive therapy was associated with improved survival $(P<0.02$ TP1, $P<0.001$ TP2). Overall survival of all patients in TP2 was significantly greater than in TP1 $(P<0.001)$, with a median survival of 15 months in TP2, whereas in TP1, the survival was only 9 months. The median 1-, 3-year survivals in TP2 (54 and 34\%, respectively) were also greater than that in TP1 (31 and 7\%, respectively). A multiple logistic regression
\end{abstract}

D.-X. Lin $(\bowtie) \cdot$ Q.-Y. Zhang

The Hepatobiliary Division, Department of General Surgery,

The First Affiliated Hospital, Wenzhou Medical College,

325000 Wenzhou, China

e-mail: 1dx566@yahoo.cn

X. Li · Q.-W. Ye · F. Lin - L.-L. Li

Department of Hepatopancreatobliliary Surgery,

Mindong Hospital of Ningde, 355000 FuAn, China analysis revealed that radical resection and adjuvant therapy were the independent predictors of overall survival.

Conclusions An aggressive approach, combining extended liver resection with thrombectomy and adjuvant therapy, leads to an improved survival in the HCC patients with PVTT.

Keywords Hepatocellular carcinoma .

Portal vein tumor thrombus - Liver resection .

Thrombectomy · Adjuvant therapy

\section{Introduction}

Hepatocellular carcinomas (HCC) are commonly developed secondary to patients with hepatitis $\mathrm{B}$ and $\mathrm{C}$ in both Africa and Asia. Recently, an increasing incidence of HCC was also reported in Western countries (El-Serag and Mason 1999). The natural history of untreated HCC is poor, especially in patients with portal vein tumor thrombus (PVTT), with a median survival of 2.7 months, whereas the survival in those without PVTT was 24.4 months (Llovet et al. 1999b). The involvement of PVTT in main portal trunk could result in portal hypertension, which would further lead to gastrointestinal bleeding, ascites, and induce multiple intrahepatic tumor dissemination and recurrence (Nagasue et al. 2001; Roayaie et al. 2002; Poon et al. 2003). The treatment of HCC with PVTT is still considered as nihilism and remains a major challenge due to poor survival rates (Poon et al. 2002; Bruix and Llovet 2002; Lee et al. 2002). However, it should be noted that some patients actually survived for a long period of time after a single tumor resection or combined with neo- and/or adjuvant therapy (Yamaoka et al. 1992; Minagawa et al. 2001; Masami et al. 2001; 
Aldrighetti et al. 2009). With the improvement of surgical techniques and perioperative care, promising results have also been achieved by using aggressive procedures to treat HCC with PVTT (Minagawa et al. 2001).

At the turn of the millennium, a reappraisal of our technical approach to HCC with PVTT was conducted at Mindong Hospital, Fujian, China, and a more aggressive treatment strategy has been adopted,which consists of extended liver resection, thrombectomy, and neo- and/or adjuvant therapy. In order to evaluate the outcomes of this shift in the treatment to HCC patients, a 10-year retrospective review of all $116 \mathrm{HCC}$ patients with PVTT admitted between 1996 and 2006 has been conduced. The results confirmed that surgical removal of the main tumor and PVTT, combining with the neo- and/or adjuvant therapy, significantly improved the survival of HCC patients with PVTT.

\section{Materials and methods}

The records of all 116 individual patients diagnosed of HCC with PVTT in Mindong Hospital, Fujian, China, were included in current study. These records, including patients' inpatient hospital and clinic charts, covered the cases of a 10-year period from January 1996 to August 2006. Of all 116 cases, two time-period-based cohorts were divided according to the treatment strategy. A total of 51 patients admitted in the first 5 years (from January 1996 to December 2000) were included in Time-Period 1 (TP1), and the remaining 65 patients were included in TimePeriod 2 (TP2). Approval for chart review was obtained from the institutional review board at MinDong Hospital, FuJian, China. Surgical mortality was considered as death occurring within 60 days of surgery. Morbidity including minor complications required no intervention, while major complications required intervention.

Preoperative assessment included medical history, physical examination, and imaging studies, including abdominal ultrasound (US), computed tomography, and magnetic resonance cholangiopancreatography (MRCP). Patients from both TPs (without exception) have been undergone triphasic liver CT scan, color Doppler US, and endoscopic examination in order to determine the size and location of HCC and PVTT in portal vein and possible complications of esophageal and cardiac varices. For patients with severe varices of the esophagus, sclerotherapy has been performed prior to the surgery in order to prevent perioperative bleeding.

A surgical operation has been performed mainly based on the volume of the future remnant liver as determined by CT volumetry (Okamoto et al. 1984) and on the removable liver volume, which was evaluated by indocyanine green retention rate at $15 \mathrm{~min}$ (ICGR15) (Takasaki et al. 1980). During the operation, an intraoperative US was conducted to determine the size and location of HCC and PVTT and the relationship between the tumor and the vascular system. The plane of liver parenchymal transection has been defined as well. It should be noted here that parenchymal transection was performed by conventional forceps-fracture method until 2003, when ultrasonic dissectors were introduced during parenchymal division, which has been proven to be able to decrease the bleeding during the operation. Central venous pressure was maintained at $5 \mathrm{~cm}$ $\mathrm{H}_{2} \mathrm{O}$ or less during the parenchymal transection phase. In order to remove the PVTT, the thrombus was resected together with the liver when the thrombus was confined within the resected liver. In cases that the PVTT protruded into the portal vein 1 to $2 \mathrm{~cm}$ beyond the resection line, the tumor thrombi were removed through the opening of the involved portal vein stump at the resection surface after the tumors were resected. The hepatoduodenal ligament was taped before the procedures and blocked when necessary. In several occasions, the PVTT could not be taken out completely from the opening of the portal vein stump at the resection surface due to the extension of PVTT into the main portal trunk and/or the opposite branch, the main portal trunk was clamped, and a $1.5-$ to $2.0-\mathrm{cm}$ longitudinal incision was made at the anterior or right wall of the portal trunk. The tumor thrombi were then under direct vision and could be removed with ease. After the removal of HCC and PVTT, another US was conducted to make certain that the tumor thrombus and the impairment had been removed completely.

Clinical follow-up after surgery including serum alfa fetoprotein (AFP) and US has been scheduled every 4 to 6 weeks during the first postoperative year and at 3 month intervals thereafter. Computed tomography or magnetic resonance imaging was performed once intrahepatic recurrence was suspected.

Adjuvant therapy included preoperative transcatheter hepatic arterial chemoembolization (neo-adjuvant therapy) and postoperative transcatheter hepatic arterial chemoembolization (adjuvant therapy). Chemotherapy regimen was as follows: IFN- $\alpha\left(5 \times 10^{6} \mathrm{U}\right)$ was administered subcutaneously on days 1,3 , and 5 of each week for 4 weeks, and 5 -FU $\left(300 \mathrm{mg} / \mathrm{m}^{2}\right)$ dispersed in lipiodol $(10$ to $20 \mathrm{ml}$ ) was then administered every 2 weeks for 4 weeks, followed by embolization using gelatin sponge particles (Gelfoam; Guangzhou), until there was near complete stasis of blood flow. This procedure was then repeated at 1-month interval depending on a patient's liver function and response to treatment. As a result, the number of chemotherapy cycles varied from 1 to 6 . 


\section{Definitions}

Terminology with respect to liver resections in this paper follows the guideline of the International HepatoPancreatico-Biliary Association (IHPBA). Resections may also be described by the Couinaud segments resected (Couinaud 1994). Wedge resection denotes a nonanatomic, nonsegmental resection of the liver surrounding the HCC to a depth of 1 to $2 \mathrm{~cm}$. The level of the PVTT (Vp) detected in preoperative imaging (Japan 2003; Llovet et al. 1999a) and postoperative pathology was classified into four categories: Vp1, PVTT in distal to second-order portal branches; Vp2, PVTT in second-order branches; Vp3, PVTT in first-order branches; and Vp4, PVTT in the main trunk and/or extended into opposite branch (Kondo et al. 2009).

\section{Statistics}

Univariate comparisons of 2 time-period groups were made with the Fisher's exact test or $\chi^{2}$ test for dichotomous covariates, and the $t$ test for continuous variables. Numerical data are expressed as the mean value \pm standard deviation (SD) unless otherwise indicated. Differences were considered significant when $P=0.05$ or less. Patient survival was calculated using the Kaplan-Meier method, and the survival curves were compared using the log-rank test. Cox proportional hazards regression modeling was used to assess the effect that independent covariates had on the dependent variable survival. Logistic regression modeling was performed to assess the impact of the covariates on the presence or absence of complications. All statistical analyses were performed using SPSS version 16.0 for Windows (SPSS, Chicago, Illinois, USA).

\section{Results}

\section{Clinical data}

Table 1 compares of the clinical data between two TPs. There was no statistically significant difference between the two TPs, except that there were a larger proportion of patients with multiple of tumors in TP2 than in TP1. It should be pointed out that the data required for the Table 1 MELD-Score calculation (serum bilirubin, INR, and serum creatinine) were obtained on admission. Due to the fact that majority of HCC patients with PVTT who suffered from virus-related hepatitis and liver cirrhosis had poor liver functions on admission, as indicated by a higher serum bilirubin value and a corresponding higher MELD-Score has been observed (Table 1). Upon admission, liver function improvement and anti-virus treatments have been given to these patients with very poor liver function. These liver functions were re-evaluated prior to the surgeries and found to be improved remarkably with much lower serum bilirubin values than the values obtained on admission (Table 1). Since only serum bilirubin data were collected 1-2 prior to the surgeries, no corresponding MELD-Scores were shown in Table 1. For patients with relatively good liver function on admission, surgeries were performed 12 days after the admission. Neo-adjuvant therapy was performed in patients with good hepatic reserve and collateral circulation around the portal trunk (9 in TP1 vs. 31 in TP2, $P<0.001)$.

\section{Disease stage}

Figure 1 compares the distribution of patients by the level of the PVTT ( $\mathrm{Vp}$ ) between 2 TPs. The majority of patients (73.8\% in TP1 and $72.3 \%$ in TP2) were diagnosed at Vp2 and Vp3. It is noticeable that more patients from TP2 were diagnosed with higher $\mathrm{Vp}$ disease $(52.3 \% \mathrm{Vp} 3$ and Vp4) than from TP $1(18.3 \% \mathrm{Vp} 3$ and Vp4), with $P<0.001$.

\section{Definitive therapy}

Types of surgical resection and the surgical and postoperative data of the both cohorts are listed in Tables 2 and 3, respectively. Of all the 116 patients, 48 patients had advanced disease (Vp3, Vp4, multiple cancer, and/or distant metastasis) and did not receive any surgery. Among the remaining 68 patients, 58 patients were explored for a potentially curative (R0) resection ( 18 in TP 1 and 40 in TP 2 , $P=0.005)$ and have undergone liver resection and thrombectomy. Another 10 patients had received palliative (R1) operations (removal of the PVTT in the main portal trunk and resection of the tumor simultaneous) for esophageal varices and ascites. Two patients from TP1 and 15 from TP2 have further undergone a concomitant other organ resection $(P=0.043)$. The majorities of operations in TP2 were hemihepatectomy or extended hemihepatectomy. The extent of liver resections and the frequency of thrombectomy were greater in TP2 than those in TP1 $(P=0.039)$.

Adjuvant therapy was received by patients with confirmed positive microscopic margins at the resection surface after the operation, multiple liver cancer, and higher $\mathrm{Vp}(\mathrm{Vp} 3$ or Vp4). There were 52 patients received chemotherapy, 9 from TP1, and 43 from TP2 $(P<0.001)$.

\section{Perioperative complications}

The overall operative mortality was low in both TPs with a total of 3 died (Table 3). One of the patients who had chronic liver disease died of liver failure after extended right hepatectomy. Another one died of massive upper 
Table 1 Comparison of the clinical data of $116 \mathrm{HCC}$ with PVTT, divided into 2 timeperiod cohorts

$M E L D$ model for end-stage liver disease

a The data are expressed as the mean value \pm standard deviation; values in bracket are the corresponding percentage;

The $P$ values are included in the bracket; $N S$ indicates that it is not statistically significant

b The data were collected on admission

c The data were collected 1 or 2 days prior to the operation

d The data were collected after operation

\begin{tabular}{|c|c|c|c|}
\hline Variables & $\begin{array}{l}\text { TP1 } \\
{[N=51,(\%)]^{\mathrm{a}}}\end{array}$ & $\begin{array}{l}\text { TP2 } \\
{[N=65,(\%)]}\end{array}$ & $\begin{array}{l}\text { Total } \\
(P \text { values })\end{array}$ \\
\hline Age (years) & $45 \pm 13$ & $44 \pm 9$ & $44 \pm 11(\mathrm{NS})$ \\
\hline Sex & & & (NS) \\
\hline Men & $43(84.3)$ & $54(88.5)$ & \\
\hline Women & $8(15.7)$ & $11(11.5)$ & \\
\hline HBsAg & & & (NS) \\
\hline Positive & $44(86.3)$ & $54(83.1)$ & \\
\hline Negative & $7(13.7)$ & $11(16.9)$ & \\
\hline $\mathrm{HCV}$ & & & (NS) \\
\hline Positive & $6(11.8)$ & $9(13.8)$ & \\
\hline Negative & $45(88.2)$ & $56(86.2)$ & \\
\hline ICG-R15 & $14.8 \pm 7.6$ & $16.1 \pm 7.9$ & $15.6 \pm 6.9(\mathrm{NS})$ \\
\hline Liver cirrhosis $^{\mathrm{d}}$ & & & (NS) \\
\hline Present & $36(70.6)$ & $47(72.3)$ & \\
\hline Absent & $15(29.4)$ & $18(27.7)$ & \\
\hline CHILD-PUGH-score ${ }^{\mathrm{c}}$ & $8 \pm 5$ & $9 \pm 4$ & (NS) \\
\hline esophageal varices ${ }^{\mathrm{b}}$ & & & (NS) \\
\hline Yes & $9(17.6)$ & $17(26.2)$ & \\
\hline No & $42(82.4)$ & $48(73.8)$ & \\
\hline MELD score ${ }^{b}$ & $27.89 \pm 1.17$ & $29.03 \pm 0.81$ & (NS) \\
\hline $\operatorname{AFP}(\mathrm{ng} / \mathrm{ml})^{\mathrm{b}}$ & $21,073 \pm 80,830$ & $19,832 \pm 79,850$ & (NS) \\
\hline Serum albumin $(\mathrm{g} / \mathrm{l})^{\mathrm{c}}$ & $35.7 \pm 4.9$ & $35.8 \pm 3.7$ & (NS) \\
\hline Serum bilirubin obtained on admission $(\mu \mathrm{mol} / \mathrm{l})^{\mathrm{b}}$ & $56.4 \pm 62.9$ & $57.6 \pm 60.9$ & (NS) \\
\hline $\begin{array}{l}\text { Serum bilirubin obtained } 1-2 \text { days prior to } \\
\text { surgeries }(\mu \mathrm{mol} / 1)^{c}\end{array}$ & $15.6 \pm 13.4$ & $16.7 \pm 11.6$ & (NS) \\
\hline No. of tumors & & & $(0.048)$ \\
\hline Solitary & $43(84.3)$ & $42(64.6)$ & \\
\hline Multiple & $8(15.7)$ & $23(35.4)$ & \\
\hline Ascites $^{\mathrm{b}}$ & & & (NS) \\
\hline Present & $10(19.6)$ & $13(20.0)$ & \\
\hline Absent & $41(80.4)$ & $52(80.0)$ & \\
\hline Hepatic vein invasion ${ }^{\mathrm{b}}$ & & & (NS) \\
\hline Negative & $43(84.3)$ & $50(76.9)$ & \\
\hline Positive & $8(15.7)$ & $15(23.1)$ & \\
\hline Neo-adjuvant therapy & 9 & 31 & $(<0.001)$ \\
\hline
\end{tabular}

gastrointestinal bleeding, and the last one died of heart failure. On the contrary, the complication rate among all the operative patients was relatively high. Complications included pleural effusion (12\%), wound infection (9\%), liver failure (6\%), upper GI bleed (5\%), pneumonia (4\%), intraabdominal abscess (3\%), Cardiac failure/ arrhythmia (2\%), postoperative bowel obstruction (2\%), bile leak (1\%), DVT/pulmonary embolus $(1 \%)$, and renal failure (1\%). None of the patients received reoperation. Two patients developed intraabdominal abscesses requiring percutaneous drainage. Multivariable analysis revealed that preoperative sclerotherapy, intraoperative drain placement, advanced age ( $>60$ years), and weight loss at presentation were associated with the development of complications (Table 4). Although it is most likely that multiple factors contributed to the development of complications, drains may increase the risk of intraabdominal abscesses and pneumonia when placed in abdominal cavities for a long time. Similarly, if the drain exits the skin in close proximity to the wound, the chance of postoperative wound infection would increase significantly. Preoperative sclerotherapy may help to safeguard against upper GI bleed.

\section{Pathology}

Tumor histology results of 68 samples have shown that there were $18 \%$ well, $53 \%$ moderately, and $29 \%$ poorly 


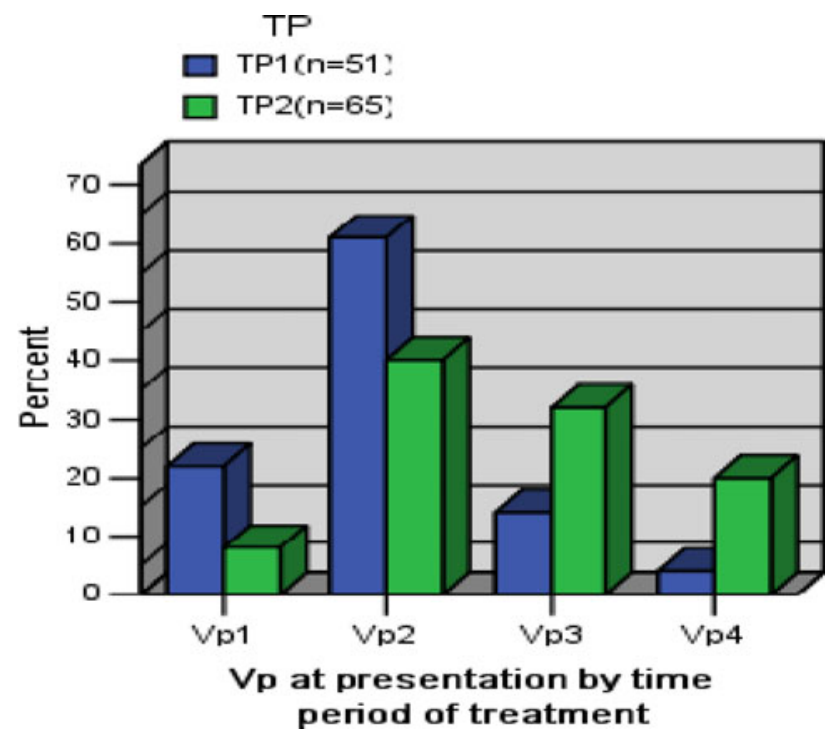

Fig. 1 Comparison of the distribution of Vps of disease at presentation between two time-periods

Table 2 Types of surgical resection of $68 \mathrm{HCC}$ with PVTT

\begin{tabular}{llll}
\hline Variables & $\begin{array}{l}\text { TP1 } \\
(N=21)\end{array}$ & $\begin{array}{l}\text { TP2 } \\
(N=47)\end{array}$ & $\begin{array}{l}\text { Total } \\
(P \text { values })\end{array}$ \\
\hline $\begin{array}{l}\text { Type of liver resection } \\
\text { Segmentectomy }\end{array}$ & 9 & 5 & $68(<0.04)$ \\
$\begin{array}{l}\text { Subsegmentectomy or wedge } \\
\text { resection }\end{array}$ & 4 & 2 & \\
Left hemihepatectomy & 2 & 10 & \\
Right hemihepatectomy & 4 & 14 & \\
$\begin{array}{l}\text { Extended left } \\
\text { hemihepatectomy }\end{array}$ & 1 & 7 & \\
$\quad$ Extended right & & & \\
hemihepatectomy & 1 & 9 & \\
Type of PVTT removal & & & \\
Liver resection alone & 16 & 27 & \\
Removal via PV stump & 4 & 15 & \\
Removal via main trunk & 1 & 5 & \\
Combined organ resection & & & \\
Partial diaphragmatic & 2 & 5 & \\
resection & & & \\
Right adrenal gland resection & 0 & 8 & \\
Splenectomy & 0 & 2 & \\
\hline
\end{tabular}

differentiated tumors. The distribution of the levels of the PVTT (Vp) was: Vp1 (22\%), Vp2 (50\%), Vp3 $(20.6 \%)$, and $\mathrm{Vp} 4(7.4 \%)$. It should be noted that the necrosis of HCC and PVTT was detected in patients undergone neo-adjuvant therapy. There was no statistically significant difference in the classification of $\mathrm{Vp}$ by $\mathrm{CT}$ preoperatively and the pathological assessment of $\mathrm{Vp}$ postoperatively in all 68 patients who had undergone resections.
Survival analysis

Figures 2, 3, and 4 compare the survival analyses results between two TPs. Patients from TP2 had better overall survival when comparing to those from TP1 $(P<0.001)$ (Fig. 2). One-, 3-year survival in TP1 was $31,7 \%$ and 54 , $34 \%$ in TP2, respectively. Median survival was 9 months in TP1 and 15 months in TP2. Patients in both time-periods had improved survival with (R0 and R1) surgical resection $(P<0.02$ in TP1; $P<0.001$ in TP2 (Fig. 3a, b). Median survival in TP1 for those undergone hepatic resection and thrombectomy and those not having surgical resection was 21 months and 6 months, respectively $(P<0.001)$. Similarly, median survival was 36 months versus 7 months in TP2 $(P<0.001)$. Analyses also showed that patients in TP2 had improved survival, when comparing the corresponding Vps to that in TP1. Differences in survival by Vp in each time-period were significant $(P$ value TP1 $<0.001$, TP2 $<0.001$; Fig. $4 \mathrm{a}, \mathrm{b}$ ). A multivariate analysis of factors revealed that only those patients undergoing liver resection combined with thrombectomy, chemotherapy, and Vp1-3 manifested a statistically significant improvement in overall survival (Table 5).

A description of the 11 long-term survivors was presented in Table 6. All patients received radical resection and chemotherapy. Ten patients suffered recurrence (mean time to recurrence, 13.3 months). The most common site of tumor recurrence was in the remnant liver. Treatment of HCC recurrence was based on the site and pattern of recurrence and the residual liver function. TACE, radiofrequency ablation (RFA), and percutaneous ethanol injection therapy (PEIT) were used for recurrent HCC.

\section{Discussion}

The management of HCC with PVTT still remained a major challenge in surgery. Barcelona Clinic Liver Cancer staging system proposed that patients with HCC and PVTT were eligible for only palliative or investigational treatments, but not for surgical intervention (Lin and Lu 1996; Minagawa et al. 2001). However, the nonsurgical management of HCC with PVTT, including systemic chemotherapy and transarterial chemoembolization, was associated with a dismal outcome of 1-year survival rate between 7 and 18\% (Okada et al. 1992; Raoul et al. 1994). On the other hand, 1-, 2-, and 3-year survival rates as high as $52.2,23.2$, and $11.6 \%$ had been reported in patients undergone radical resection including hepatectomy and thrombectomy, which was originally conducted to prevent potential tumor rupture and esophageal variceal bleeding (Yamaoka et al. 1992). Ohkubo et al (2000) and Masami et al (2001) had also reported similar outcomes with 
Table 3 Comparison of surgical and postoperative data of 68 HCC with PVTT

\begin{tabular}{llll}
\hline Variables & TP1 $(N=21)$ & TP2 $(N=47)$ & Total $(P$ values $)$ \\
\hline Average overall operative time (min) & 167 & 14 & 155 (NS) \\
Average intraoperative blood loss (ml) & 1,210 & 1,310 & 1,270 (NS) \\
Required blood transfusion & 2 & 4 & 6 (NS) \\
Average occlusion time of the hepatic inflow (min) & 11 & 12.5 & 11.8 (NS) \\
Adjuvant therapy & 9 & 43 & $(<0.001)$ \\
Operative mortality & $4.8 \%$ & $4.3 \%$ & $4.4 \%(\mathrm{NS})$ \\
Postoperative complication & $42.9 \%$ & $44.7 \%$ & $45.6 \%(\mathrm{NS})$ \\
Mean length of stay (days) & 12.7 & 9.8 & 10.7 (NS) \\
\hline
\end{tabular}

NS not significant

Table 4 Factors predictive of complications following surgery for HCC with PVTT: multivariate analysis

\begin{tabular}{|c|c|c|c|}
\hline Covariates & $P$ value & OR & $95 \% \mathrm{CI}$ \\
\hline Time-period $(1,2)$ & 0.96 & & \\
\hline Preoperative TACE (yes, no) & 0.90 & & \\
\hline Combined organ resection (yes, no) & 0.81 & & \\
\hline Sex (male, female) & 0.70 & & \\
\hline Estimated blood loss $(<1,000 \mathrm{ml},>1,000 \mathrm{ml})$ & 0.61 & & \\
\hline Ascites (yes, no) & 0.55 & & \\
\hline Operating time $(<2 \mathrm{~h},>2 \mathrm{~h})$ & 0.27 & & \\
\hline Drain (yes, no) & 0.001 & 79.13 & $6.53-1,040$ \\
\hline Preoperative sclerotherapy (yes, no) & 0.002 & 61.73 & $4.42-750$ \\
\hline Age $(<60,>60$ years $)$ & 0.027 & 18.63 & $2.86-196$ \\
\hline Weight loss at presentation (yes, no) & 0.045 & 11.50 & $1.34-135$ \\
\hline
\end{tabular}

Fig. 2 Comparison of overall survival between 2 timeperiods: Kaplan-Meier curve

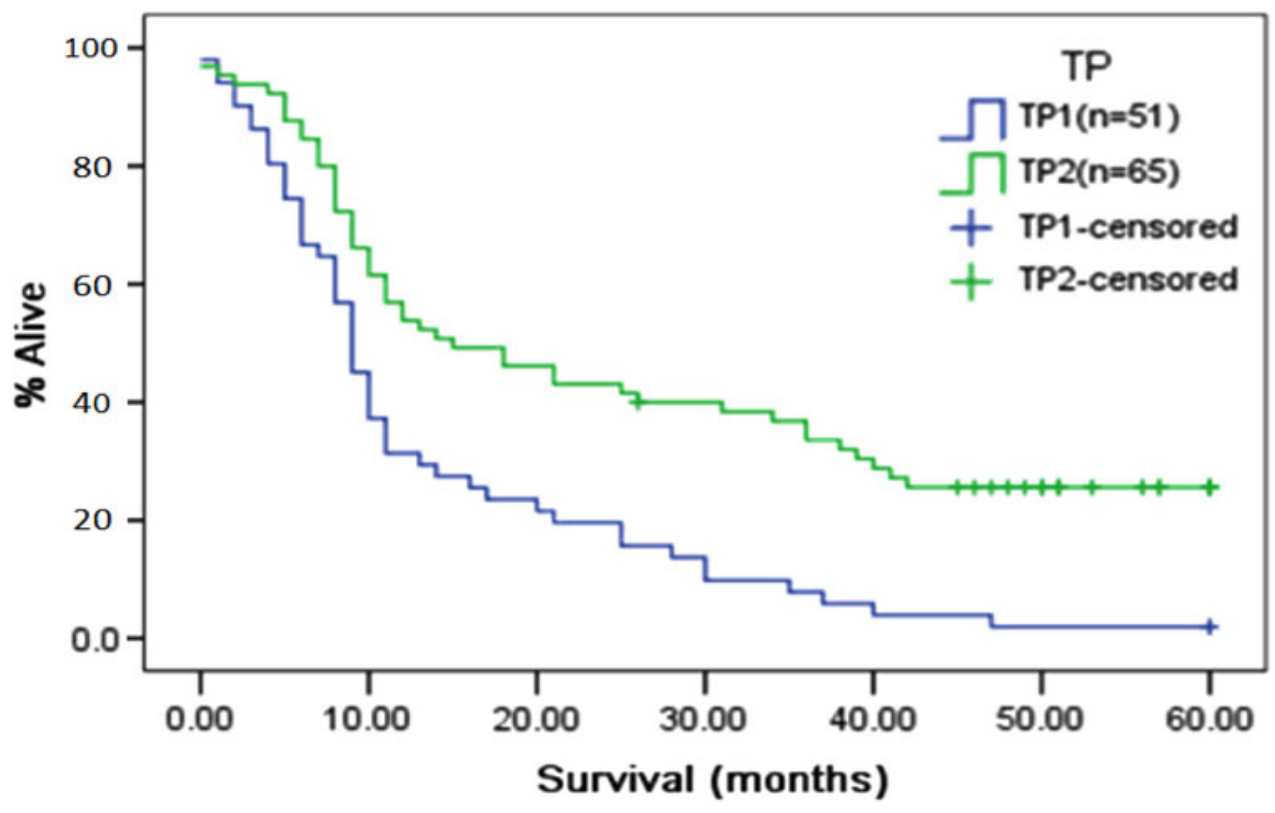

aggressive therapies. These results led to a reappraisal of our aggressive approach to HCC patients with PVTT at the turn of the century. In this paper, we compared the aggressive management and survival outcomes of 2 distinct approaches applied to this disease over the past 10 years. Our results have shown that both the overall survival and 
Fig. 3 Effect of liver resection combined with thrombectomy on patients' survival in TP1 (a) and TP2 (b)
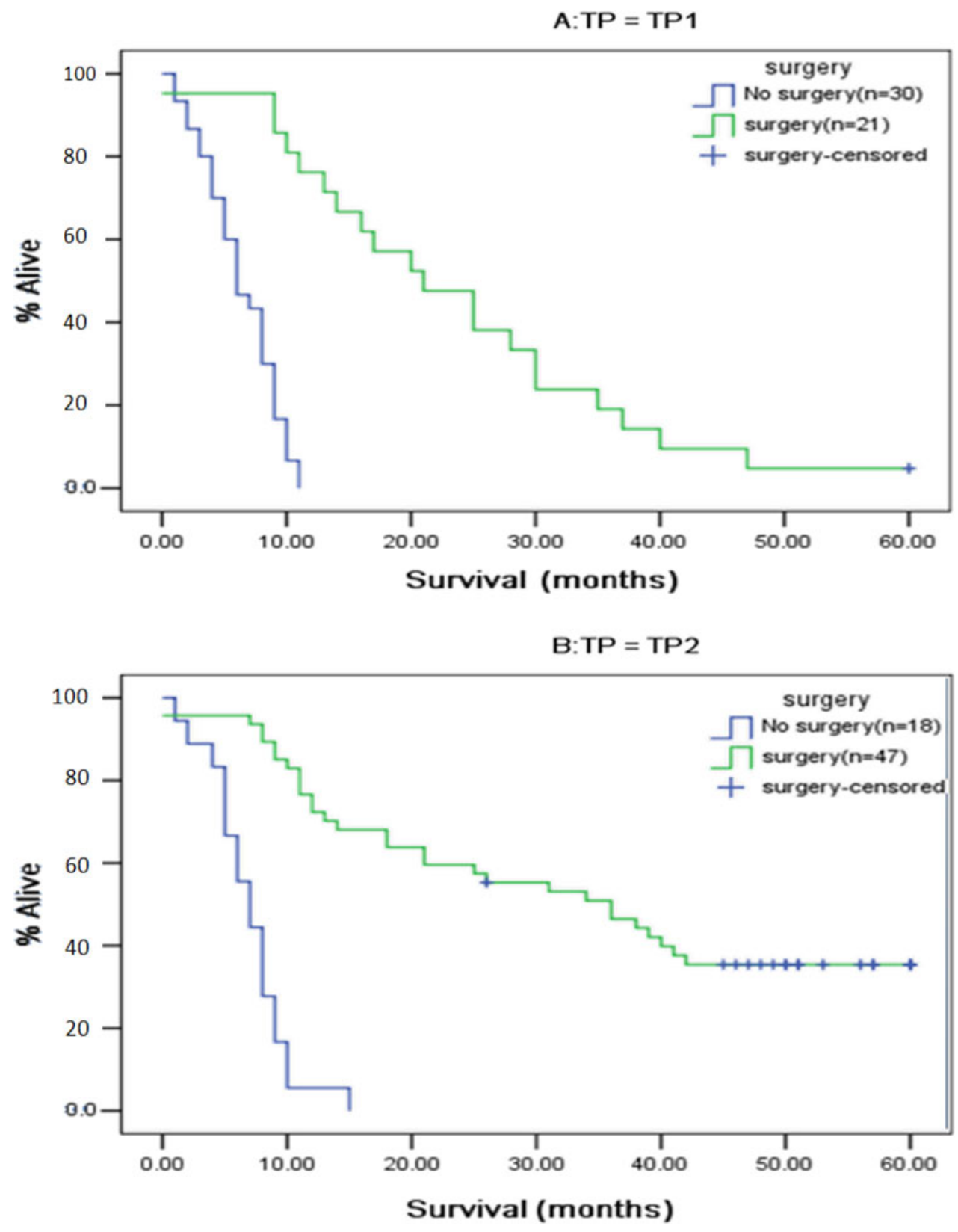

the median 1-, 3-year survivals of all patients in TP2 were significantly greater than that in TP1, despite the fact that patients in TP2 had a larger proportion of multiple of tumors and higher $\mathrm{Vp}$ disease.

Current study provided solid evidence that radical resections could not only be performed safely with minimal mortality $(4.4 \%)$ but greatly increase patients' 1-, 3-year postoperative survival rates. It was worth pointing out here that multivariate analyses had ruled out the radical resection as the contribution factor to the high postoperative complications rate $(45.6 \%)$. Multivariate analyses demonstrated that the most important factor determining longterm survival was liver resection with thrombectomy
$(P<0.001)$. Surgical procedure was the only modality that had the potential to completely remove the gross tumor from the liver parenchyma and portal vein as well as minimize residual tumor burden (Llovet et al. 1999b). The benefits of tumor and thrombus resection en bloc or hepatectomy plus thrombectomy could be summarized as follows: (1) decrease in portal vein pressure and prevention of intractable ascites and esophageal varices bleeding; (2) recovery of blood flow of portal vein and improvement of liver function; (3) reducing the tumor burden and increasing the efficacy of postoperative multimodality treatments such as TACE, PVI, and HAI; and (4) improvement of quality of life and survival rate of the patients (Tazawa 
Fig. 4 Comparison of patients' survival between Vps in TP1 (a) and TP2 (b)
$A: T P=$ TP1

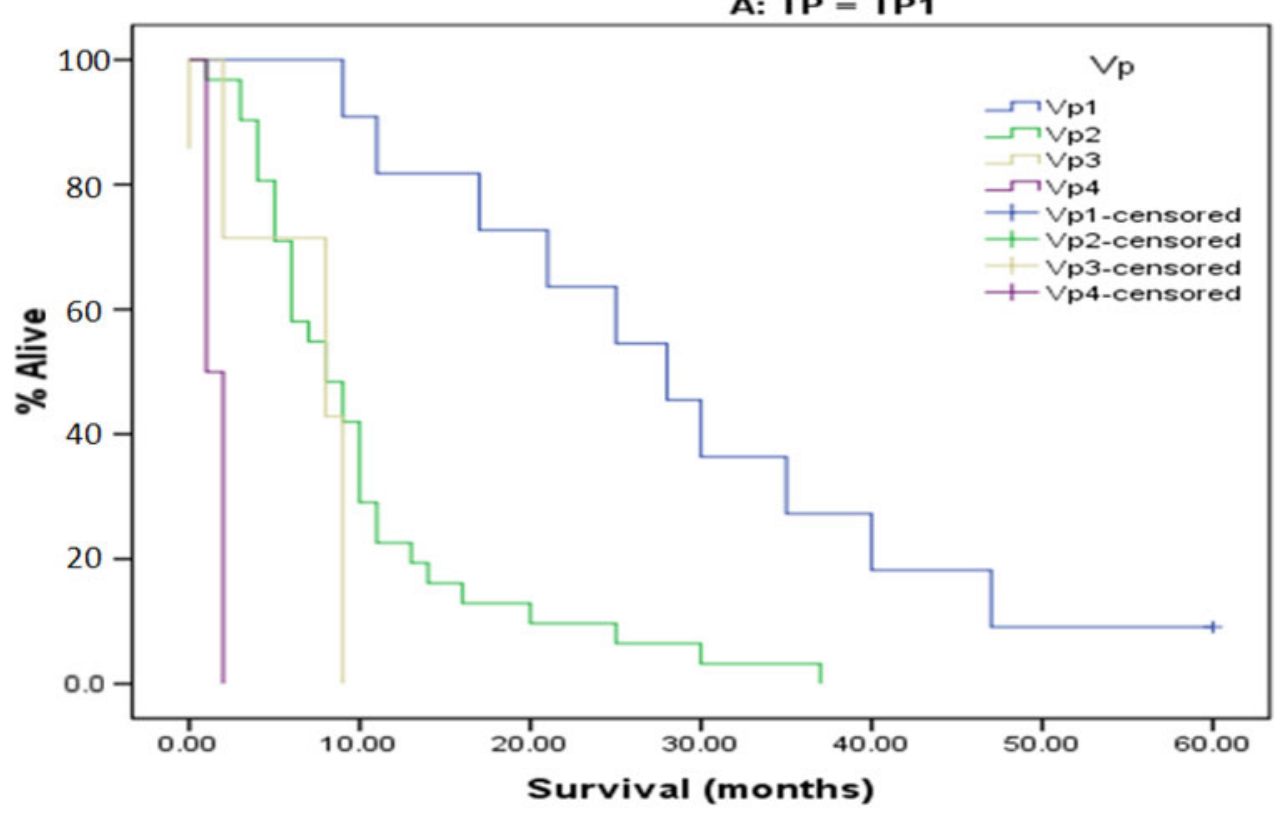

B: $\mathbf{T P}=\mathbf{T P 2}$

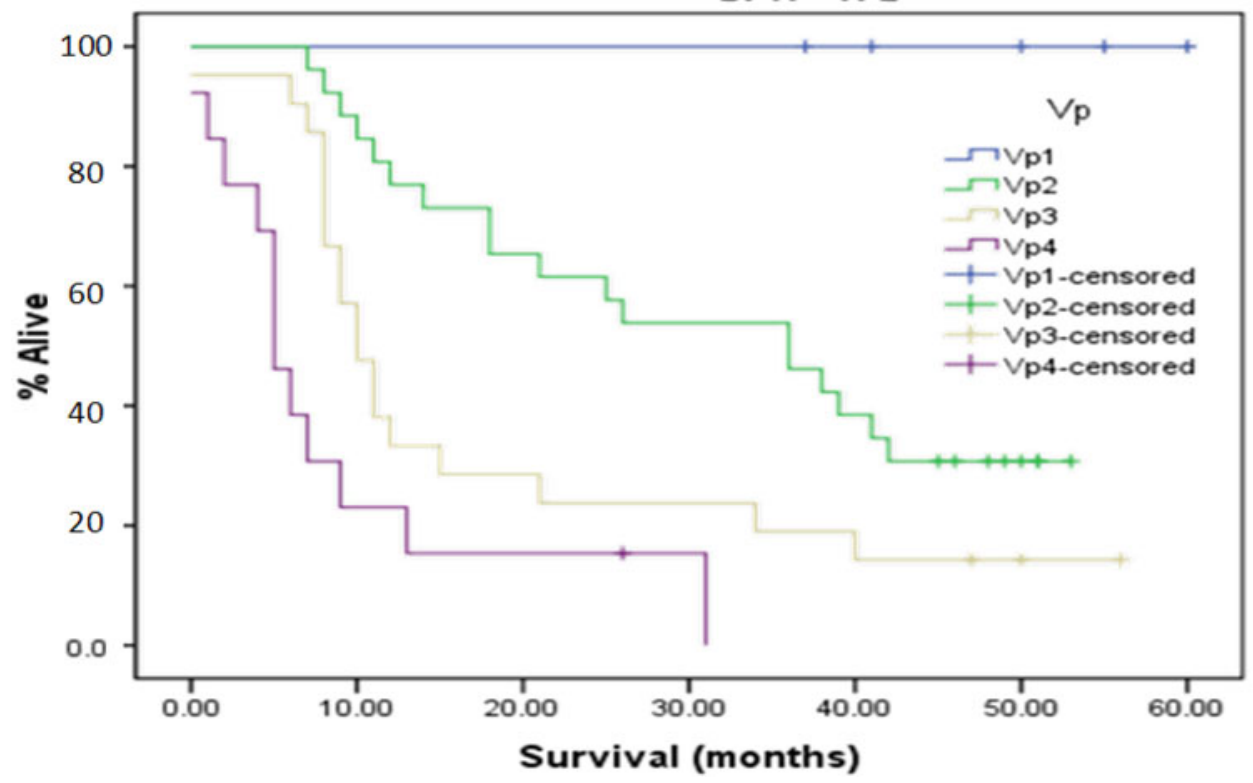

et al. 2001; Inoue et al. 2004). Therefore, surgical resection was an effective therapy that could help to improve the survival for HCC patients with PVTT.

Cox's multivariate analysis showed that the level of the PVTT (Vp) was an independent survival predictor for HCC patients with PVTT. The postoperative prognosis of patients varied with the different level of $\mathrm{Vp}$ (Chen et al. 2006). The 1- and 3-year survival rates for patients with Vp1-3 and Vp4 were 52.1, 16.0\%, and 33.1, 0\%, respectively. All patients with Vp4 died from recurrent HCC within 400 days after surgery, while some patients with Vp1, 2, or 3 obtained longer survival. These data suggests that patients without Vp4 are encouraged to undergo surgery in order to obtain longer survival. On the other hand, surgery is not recommended to be the first choice of treatment for patients with Vp4 unless it is an emergent case with impending rupture of esophagogastric varices due to portal hypertension or acute liver failure caused by PVTT (Llovet et al. 1999a). Resection in such patients should be re-evaluated when more effective adjuvant treatments are available. It should be noted that most recently, liver transplantation has been recommended to be an alternative therapeutic for patients with HCC and PVTT (Xu et al. 2004; Sotiropoulos et al. 2008). Giving the fact 
Table 5 Factors predictive of long-term outcome

\begin{tabular}{|c|c|c|c|c|}
\hline \multirow[t]{2}{*}{ Covariates } & \multirow{2}{*}{$\begin{array}{l}\text { Univariate } \\
\text { analysis } \\
\text { Log-rank: } \\
P \text { value }\end{array}$} & \multicolumn{3}{|c|}{ Multivariate analysis } \\
\hline & & $P$ value & HR & $95 \% \mathrm{CI}$ \\
\hline Radical resection & 0.0001 & 0.001 & 0.28 & $0.14-0.59$ \\
\hline $\mathrm{Vp}(\mathrm{Vp} 1-3, \mathrm{Vp} 4)$ & 0.008 & 0.002 & 2.34 & $1.29-5.00$ \\
\hline Chemotherapy & 0.002 & 0.05 & 2.29 & $0.99-4.72$ \\
\hline Weight loss at presentation & 0.0001 & 0.28 & 1.60 & $0.82-3.17$ \\
\hline No. of tumors & 0.003 & 0.54 & 0.77 & $0.61-1.23$ \\
\hline Time-period & 0.001 & 0.61 & 0.81 & $0.38-1.54$ \\
\hline ICG-R $_{15}$ & 0.03 & 0.87 & 0.93 & $0.47-1.87$ \\
\hline Combined organ resection & 0.11 & & & \\
\hline Liver cirrhosis & 0.77 & & & \\
\hline Esophageal varices & 0.16 & & & \\
\hline Ascites & 0.43 & & & \\
\hline Blood transfusion & 0.07 & & & \\
\hline Estimated blood loss & 0.93 & & & \\
\hline Preoperative sclerotherapy & 0.81 & & & \\
\hline $\operatorname{AFP}(\mathrm{ng} / \mathrm{ml})(\leq 25,>25)$ & 0.87 & & & \\
\hline $\mathrm{HBsAg}$ & 0.76 & & & \\
\hline Age $(<60,>60)$ & 0.91 & & & \\
\hline
\end{tabular}

that current liver transplantation is associated with high recurrence rate, high treatment cost and serious shortage of organ donors, HCC patients with PVTT, especially those with Vp3-4, were not recommended to undergo liver transplantation (Van Thiel et al. 1998; Fan et al. 2000; Iwatsuki et al. 2000; Frilling et al. 2001).

The results from current study also showed that surgical removal of the main tumor and PVTT, combining with the neo- and/or adjuvant therapy, significantly improved the survival of HCC patients with PVTT. The pre- and/or postoperative chemotherapy was more frequently received in TP2 than in TP1, which in part contributed to improve the survival of patients in TP2. It was reported that neo-adjuvant therapy could prevent the growth of HCC into the portal vein and was effective in the regression of portal thrombus (Chung et al. 1995; Vetter et al. 1991). IFN- $\alpha$ had a synergistic effect with 5-FU and was able to enhance the antitumoral efficacy of chemotherapy (Oie et al. 2006; Koike et al. 2006). Accumulation of iodized oil in PVTT, not only in HCC nodules, was seen in many patients, and necrosis of PVTT was detected by pathologic examination (Minagawa et al. 2001). Because hepatic artery supply fed both the HCC and the PVTT, and the residual microscopic tumor and micrometastases could be existed in liver, adjuvant therapy had been used after liver resection combined with PVTT removal (Liang et al. 2008; Peng et al. 2009). This approach has been proven to be able to prolong patient's survival (Fan et al. 2003).

In summary, a remarkable improvement has been achieved in the outcome of the treatment of patients with HCC and PVTT over the past 5 years, after a shift to a more aggressive approach. Multivariate examination accounting for the time-period in which treatment performed revealed that the most important factor determining long-term survival was whether the patient underwent an aggressive liver resection with thrombectomy and chemotherapy. On the other hand, even in the most recent timeperiod, a maximal 3-year survival rate of only $34 \%$ has been achieved. Therefore, it is essential that novel adjuvant therapies should be developed and evaluated for these patients.

Table 6 Actual long-term survivors: Following surgery for HCC with PVTT

\begin{tabular}{llllll}
\hline TP & Age/sex & Vp & Surgical resection & Adjuvant therapy & Survival/status \\
\hline TP1 & 48 years/M & Vp1 & Yes & Pre- and postoperative TACE & 4 years 10 months DOD \\
TP1 & 56 years/M & Vp2 & Yes & Preoperative TACE & 5 years 9 months DOD \\
TP1 & 59 years/M & Vp3 & Yes & Pre- and postoperative TACE & 4 years 2 months DOD \\
TP2 & 42 years/M & Vp3 & Yes & Preoperative TACE & 3 years 6 months NED \\
TP2 & 64 years/F & Vp3 & Yes & Postoperative TACE & 4 years 1 months DOD \\
TP2 & 43 years/M & Vp2 & Yes & Preoperative TACE & 4 years 9 months DOD \\
TP2 & 57 years/M & Vp3 & Yes & Preoperative TACE & 8 years 5 months NED \\
TP2 & 65 years/M & Vp1 & Yes & Preoperative TACE & 5 years 9 months NED \\
TP2 & 57 years/M & Vp3 & Yes & Pre- and postoperative TACE & 3 years 7 months DOD \\
TP2 & 33 years/M & Vp1 & Yes & Postoperative TACE & 4 years 1 months DOD \\
TP2 & 45 years/M & Vp2 & Yes & Preoperative TACE & 7 years 3 months NED \\
\hline
\end{tabular}

$T A C E$ transcatheter hepatic arterial chemoembolization, DOD dead of disease, $N E D$ no evidence of disease 


\section{Conflict of interest statement None.}

Open Access This article is distributed under the terms of the Creative Commons Attribution Noncommercial License which permits any noncommercial use, distribution, and reproduction in any medium, provided the original author(s) and source are credited.

\section{References}

Aldrighetti L, Pulitano C, Catena M, Arru M, Guzzetti E, Halliday J, Ferla G (2009) Liver resection with portal vein thrombectomy for hepatocellular carcinoma with vascular invasion. Ann Surg Oncol 16(5):1254. doi:10.1245/s10434-009-0383-y

Bruix J, Llovet JM (2002) Prognostic prediction and treatment strategy in hepatocellular carcinoma. Hepatology 35(3):519524. doi:10.1053/jhep.2002.32089

Chen XP, Qiu FZ, Wu ZD, Zhang ZW, Huang ZY, Chen YF, Zhang BX, He SQ, Zhang WG (2006) Effects of location and extension of portal vein tumor thrombus on long-term outcomes of surgical treatment for hepatocellular carcinoma. Ann Surg Oncol 13(7):940-946. doi:10.1245/ASO.2006.08.007

Chung JW, Park JH, Han JK, Choi BI, Han MC (1995) Hepatocellular carcinoma and portal vein invasion: results of treatment with transcatheter oily chemoembolization. AJR Am J Roentgenol 165(2):315-321

Couinaud C (1994) Intrahepatic anatomy. Application to liver transplantation. Ann Radiol (Paris) 37(5):323-333

El-Serag HB, Mason AC (1999) Rising incidence of hepatocellular carcinoma in the United States. N Engl J Med 340(10):745-750

Fan ST, Cheung ST, Lo CM (2000) Indications for liver transplantation in patients with chronic hepatitis $\mathrm{B}$ and $\mathrm{C}$ virus infection and hepatocellular carcinoma. J Gastroenterol Hepatol 15(SupplE):181-186

Fan J, Wu ZQ, Zhou J, Qiu SJ, Shi YH, Chen RX, Tang ZY (2003) Hepatocellular carcinoma associated with tumor thrombosis in the portal vein: the effects of different treatments. Hepatobiliary Pancreat Dis Int 2(4):513-519

Frilling A, Malago M, Broelsch CE (2001) Current status of liver transplantation for treatment of hepatocellular carcinoma. Dig Dis 19(4):333-337

Inoue K, Nakamura T, Kinoshita T, Konishi M, Nakagohri T, Oda T, Takahashi S, Gotohda N, Hayashi T, Nawano S (2004) Volume reduction surgery for advanced hepatocellular carcinoma. J Cancer Res Clin Oncol 130(6):362-366. doi:10.1007/s00432004-0566-7

Iwatsuki S, Dvorchik I, Marsh JW, Madariaga JR, Carr B, Fung JJ, Starzl TE (2000) Liver transplantation for hepatocellular carcinoma: a proposal of a prognostic scoring system. J Am Coll Surg 191(4):389-394. doi:S1072-7515(00)00688-8

Japan LCSGo (2003) General rules for the clinical and pathological study of primary liver cancer, 2 English edn. Kanehara, Tokyo

Koike K, Takaki A, Tatsukawa M, Suzuki M, Shiraha H, Iwasaki Y, Sakaguchi K, Shiratori Y (2006) Combination of 5-FU and IFNalpha enhances IFN signaling pathway and caspase-8 activity, resulting in marked apoptosis in hepatoma cell lines. Int J Oncol 29(5):1253-1261

Kondo K, Chijiiwa K, Kai M, Otani K, Nagaike K, Ohuchida J, Hiyoshi M, Nagano M (2009) Surgical strategy for hepatocellular carcinoma patients with portal vein tumor thrombus based on prognostic factors. J Gastrointest Surg 13(6):1078-1083. doi: 10.1007/s11605-009-0854-2

Lee WC, Jeng LB, Chen MF (2002) Estimation of prognosis after hepatectomy for hepatocellular carcinoma. Br J Surg 89(3):311316. doi:10.1046/j.0007-1323.2001.02034.x
Liang LJ, Hu WJ, Yin XY, Zhou Q, Peng BG, Li DM, Lu MD (2008) Adjuvant intraportal venous chemotherapy for patients with hepatocellular carcinoma and portal vein tumor thrombi following hepatectomy plus portal thrombectomy. World J Surg 32(4):627-631. doi:10.1007/s00268-007-9364-0

Lin JP, Lu DS (1996) Early enhancement of tumor thrombus in the portal vein on two-phase helical CT. J Comput Assist Tomogr 20(4):653-655

Llovet JM, Bru C, Bruix J (1999a) Prognosis of hepatocellular carcinoma: the BCLC staging classification. Semin Liver Dis 19(3):329-338

Llovet JM, Bustamante J, Castells A, Vilana R, Ayuso Mdel C, Sala M, Bru C, Rodes J, Bruix J (1999b) Natural history of untreated nonsurgical hepatocellular carcinoma: rationale for the design and evaluation of therapeutic trials. Hepatology 29(1):62-67. doi:10.1002/hep.510290145

Masami M, Masatoshi M, Tadatoshi T et al (2001) Selection criteria for hepatectomy in patients with hepatocellular carcinoma and portal vein tumor thrombus. Ann Surg 233:379-384

Minagawa M, Makuuchi M, Takayama T, Ohtomo K (2001) Selection criteria for hepatectomy in patients with hepatocellular carcinoma and portal vein tumor thrombus. Ann Surg 233(3):379-384

Nagasue N, Ono T, Yamanoi A, Kohno H, El-Assal ON, Taniura H, Uchida M (2001) Prognostic factors and survival after hepatic resection for hepatocellular carcinoma without cirrhosis. Br J Surg 88(4):515-522. doi:10.1046/j.1365-2168.2001.01732.x

Ohkubo T, Yamamoto J, Sugawara Y, Shimada K, Yamasaki S, Makuuchi M, Kosuge T (2000) Surgical results for hepatocellular carcinoma with macroscopic portal vein tumor thrombosis. J Am Coll Surg 191(6):657-660. doi:S1072-7515(00)00740-7

Oie S, Ono M, Yano H, Maruyama Y, Terada T, Yamada Y, Ueno T, Kojiro M, Hirano K, Kuwano M (2006) The up-regulation of type I interferon receptor gene plays a key role in hepatocellular carcinoma cells in the synergistic antiproliferative effect by 5-fluorouracil and interferon-alpha. Int J Oncol 29(6):1469-1478

Okada S, Okazaki N, Nose H, Yoshimori M, Aoki K (1992) Prognostic factors in patients with hepatocellular carcinoma receiving systemic chemotherapy. Hepatology 16(1):112-117. doi:S0270913992001733

Okamoto E, Kyo A, Yamanaka N, Tanaka N, Kuwata K (1984) Prediction of the safe limits of hepatectomy by combined volumetric and functional measurements in patients with impaired hepatic function. Surgery 95(5):586-592

Peng BG, He Q, Li JP, Zhou F (2009) Adjuvant transcatheter arterial chemoembolization improves efficacy of hepatectomy for patients with hepatocellular carcinoma and portal vein tumor thrombus. Am J Surg 198(3):313-318. doi:10.1016/j.amjsurg. 2008.09.026

Poon RT, Fan ST, Lo CM, Liu CL, Wong J (2002) Long-term survival and pattern of recurrence after resection of small hepatocellular carcinoma in patients with preserved liver function: implications for a strategy of salvage transplantation. Ann Surg 235(3):373-382

Poon RT, Fan ST, Ng IO, Wong J (2003) Prognosis after hepatic resection for stage IVA hepatocellular carcinoma: a need for reclassification. Ann Surg 237(3):376-383. doi:10.1097/01.SLA. 0000055224.68432.80

Raoul JL, Guyader D, Bretagne JF, Duvauferrier R, Bourguet P, Bekhechi D, Deugnier YM, Gosselin M (1994) Randomized controlled trial for hepatocellular carcinoma with portal vein thrombosis: intra-arterial iodine-131-iodized oil versus medical support. J Nucl Med 35(11):1782-1787

Roayaie S, Frischer JS, Emre SH, Fishbein TM, Sheiner PA, Sung M, Miller CM, Schwartz ME (2002) Long-term results with multimodal adjuvant therapy and liver transplantation for the 
treatment of hepatocellular carcinomas larger than 5 centimeters. Ann Surg 235(4):533-539

Sotiropoulos GC, Radtke A, Schmitz KJ, Molmenti EP, Schroeder T, Saner FH, Baba HA, Fouzas I, Broelsch CE, Malago M, Lang H (2008) Liver transplantation in the setting of hepatocellular carcinoma and portal vein thrombosis: a challenging dilemma? Dig Dis Sci 53(7):1994-1999. doi:10.1007/s10620-007-0099-4

Takasaki T, Kobayashi S, Suzuki S, Muto H, Marada M, Yamana Y, Nagaoka T (1980) Predetermining postoperative hepatic function for hepatectomies. Int Surg 65(4):309-313

Tazawa J, Maeda M, Sakai Y, Yamane M, Ohbayashi H, Kakinuma S, Miyasaka Y, Nagayama K, Enomoto N, Sato C (2001) Radiation therapy in combination with transcatheter arterial chemoembolization for hepatocellular carcinoma with extensive portal vein involvement. J Gastroenterol Hepatol 16(6):660-665

Van Thiel DH, Colantoni A, De Maria N (1998) Liver transplantation for hepatocellular carcinoma? Hepatogastroenterology 45(24): 1944-1949
Vetter D, Wenger JJ, Bergier JM, Doffoel M, Bockel R (1991) Transcatheter oily chemoembolization in the management of advanced hepatocellular carcinoma in cirrhosis: results of a Western comparative study in 60 patients. Hepatology 13(3):427-433

Xu X, Zheng SS, Liang TB, Wang WL, Jin J, Shen Y, Wu J, Yu J (2004) Orthotopic liver transplantation for patients with hepatocellular carcinoma complicated by portal vein tumor thrombi. Hepatobiliary Pancreat Dis Int 3(3):341-344

Yamaoka Y, Kumada K, Ino K, Takayasu T, Shimahara Y, Mori K, Tanaka A, Morimoto T, Taki Y, Washida M et al (1992) Liver resection for hepatocellular carcinoma (HCC) with direct removal of tumor thrombi in the main portal vein. World J Surg 16(6):1172-1176 